\title{
LONG-TERM SPOT PHOTOMETRY AND DIFFERENTIAL ROTATION OF THE SPOTTED FLARE STARS EV Lac \\ AND BY Dra
}

\author{
K.P. PANOV \\ Department of Astronomy and National Astronomical \\ Observatory, Bulgarian Academy of Sciences, \\ 72 Trakya Blvd, 1784 Sofia, Bulgaria.
}

Ev Lac $=$ Gliese $873(\mathrm{dM} 4.5 \mathrm{e})$ is a well-known flare star with hydrogen Balmer lines as well as with Ca II lines in emission. Rotational modulation by starspots has been discovered by Pettersen (1980) who determined the photometric (rotational) period $\mathrm{P}=4.378 \mathrm{~d}$. Spot photometry of EV Lac was obtained with the $60 \mathrm{~cm}$ telescope of the Bulgarian National Astronomical Observatory in the ycars 1983-87 and 198991. For these years (except for 1989 and 1990) light curves are available in the $\mathrm{V}$ and B bands. From the BNAO-data and the data published by Pettersen (1980) a new ephemeris

$$
J D(\text { min.light })=2445906.369+4.3734 \cdot E
$$

has been derived which shows a slight revision of the period.

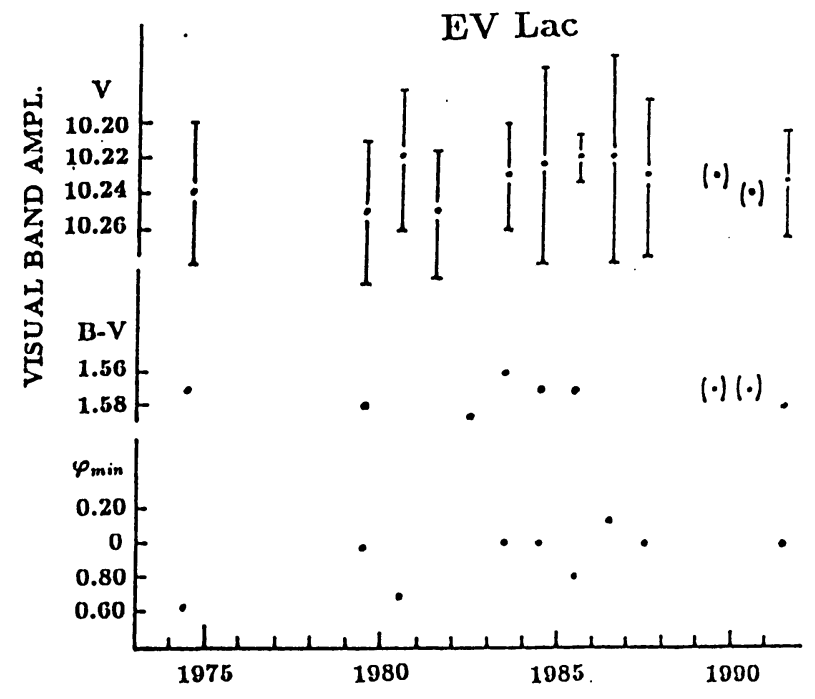

Fig. 1. Long-term spot behaviour for EV Lac. Bars denote the range of amplitude in the visual band, due to rotational modulation.

In Fig. 1 the mean visual magnitude of EV Lac and the range of the amplitude is plotted for the respective year, together with the B-V colour and the phases of the minimum light. Published data have been taken into account and supplemented 
with this study. The bars in the top pannel indicate the peak-to-peak amplitude of light variations. The amplitude of the rotational modulation is strongly variable (e.g. $0.03 \mathrm{mag}$ in 1985, and $0.12 \mathrm{mag}$ in 1986) and this is indicative for the development and redistribution of spots. But an active longitude seems to persist, thus it is possible to determine a mean rotational period. Mavridis and Avgoloupis (1986) have claimed the existence of a 5-year activity cycle in EV Lac, visible in mean light variations of the quiet star and in the level of flare activity. Fig. 1 provides no support for a stellar cycle in EV Lac (actually there are no long-term variations of mean light and colour), however, flare activity, which is not considered here, could be an independent indicator. In the bottom pannel of Fig. 1 shifts are seen in the phase of minimum light, which may be due to a latitudinal migration of spots and differential rotation. From the phase shifts the rate of differential rotation could be obtained as $\Delta P / P=\Delta \Omega / \Omega \approx 2 \cdot 10^{-3}$. Since this estimate is based on only a few data sets it should be regarded with caution.

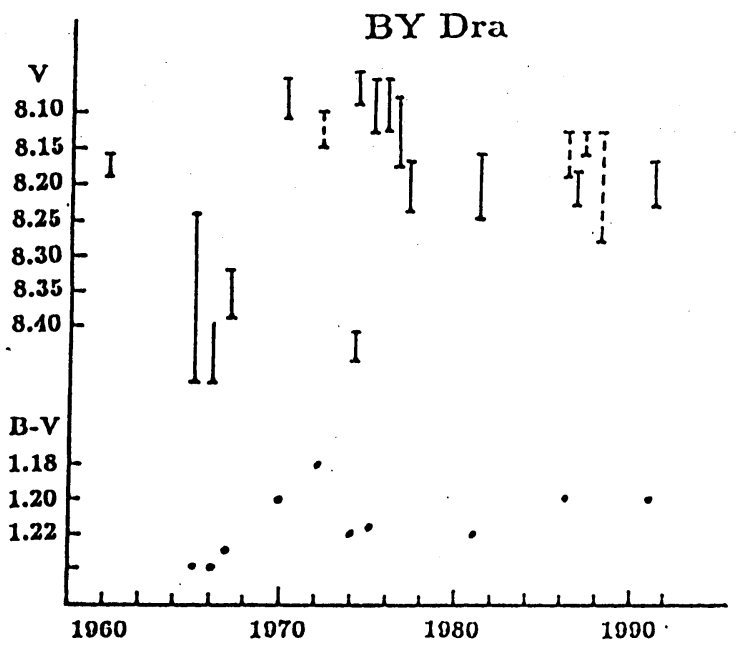

Fig.2. Long-term variability of BY Dra. Bars denote the range of the amplitude.

BY Dra $=$ Gliese $719(\mathrm{dMOe}+\mathrm{dMe})$ is a double-lined spectroscopic binary with an orbital period of $5.975 \mathrm{~d}$ (Vogt and Fekel, 1979). Chugainov (1966) observed light variations due to rotational modulation by starspots and determined the rotational period $\mathrm{P}=3.836 \mathrm{~d}$. Chugainov (1973) suggested an 8-9-year spot cycle visible in the mean light variations and also in variations of colour and period. Phillips and Hartmann (1978) revealed the existence of light variations since the early 1900's indicating a possible cycle of 50 - 60 years. Vogt (1981) found evidence for a latitudinal migration of the spots and derived the first estimate of the latitudinal shear for BY Dra, which was found to be about the same valuc as for the Sun. Spot photometry of BY Dra was obtained at BNAO in 1986-88 and in 1991, and light curves were constructed with the period of $3.836 \mathrm{~d}$. In Fig. 2 the range of the amplitude in the visual band is plotted for the respective year together with the $\mathrm{B}-\mathrm{V}$ colour. 
Data were taken from different publications and supplemented with this study. The amplitude of light variations due to spot modulation is strongly variable (e.g. 0.03 mag in 1987 and $0.15 \mathrm{mag}$ in 1988) as seen from Fig. 2. Also the long-term variation of the mean light and colour is obvious. This, however, is mainly due to the spot development during 1965-73, which could be an episode of activity. The mean light shows little changes during 1977-91 and there is no compelling evidence for a cycle.

Some of the light curves, which have been obtained at BNAO in different years, show phase-shifts, if plotted with the $3.836 \mathrm{~d}$ period. As in the case of EV Lac it seems possible that the period changes are caused by a latitudinal migration of spots and under the influence of differential rotation. From these phase-shifts with respect to the epoch JD 2446659.45 (min light), the rate of differential rotation of BY Dra can be estimated to be $\triangle P / P \approx 2 \cdot 10^{-3}$. Although the obtained rates of differential rotation for both EV Lac and BY Dra are only rough estimates, they seem to be much smaller (by two orders of magnitude) than the respective value for the Sun. On the other hand these estimates seem to agree well with the respective values, obtained for other active late-type stars.

This work has been supported by grant F-108 of the Bulgarian Ministry of Education and Science.

\section{References}

Chugainov, P.F.: 1966, IAU Inf. Bull. Var. Stars 122.

Chugainov, P.F.: 1973, Izv. Krym. Astrofiz. Obs. 48, 3.

Mavridis, L.N., Avgoloupis, S.: 1986, Astron. Astrophys. 154, 171.

Pettersen, B.R.: 1980, Astron. J. 85, 871.

Phillips, M.J., Hartmann, L.: 1978, A strophys. J. 224, 182.

Vogt, S.S., Fekel, F.: 1979, A strophys. J. 234, 958.

Vogt, S.S.: 1981, Astrophys. J. 250, 327. 